\title{
Can we please reconsider intranasal injections?
}

\author{
William Moss ${ }^{1}$ \\ ${ }^{1}$ Chapters
}

September 11, 2020

\begin{abstract}
Introduction: Intranasal steroid injections were once routinely performed for refractory allergy patients worldwide. Due to concerns about visual complications, the procedure is very rarely performed today. Nonetheless, many studies evaluating the technique advocate for its use. Methods: A narrative review of the history of intranasal steroid injections was performed. Additionally, the analogous experiences of non-otolaryngology providers with steroid injection treatments were assessed. Results: Many non-otolaryngology providers have had similar, devastating thromboembolic complications from particulate steroid injections. In other fields, a switch to non-particulate steroid formulations (e.g. dexamethasone) appears to avoid the risk of thromboembolic events entirely without sacrificing effectiveness. Conclusion: The otolaryngologists' aversion to intranasal injection treatments may not be evidence-based. Intranasal injection treatments with non-particulate steroids as well as other medications merit consideration.
\end{abstract}

\section{Main Document}

The otolaryngologist's non-surgical toolbox for treating sinonasal allergies is practically empty. Allergy patients have nearly universally failed topical steroids and frontline oral medications prior to being evaluated by a specialist. For those who don't meet criteria for surgery, the options are underwhelming; Switch to another topical formulation? Pass it off to an allergist? In the 1950's and '60's, such patients routinely underwent a different treatment-an intranasal steroid injection (Figure I ). This simple, inexpensive, and historically extremely effective intervention was once standard of care in otolaryngology clinics across the world. Typically performed every few months, systemic complications, such as elevated serum glucose, were transient and clinically-insignificant. That was before a series of now infamous case reports appeared describing blindness following intranasal injections. The intervention rapidly fell out of favor, and has remained sidelined ever since.

Multiple prior reviews have analyzed these safety concerns, ultimately making an argument in support of intranasal steroid injections. ${ }^{1,2}$ A review from 2015 described significant deviations from the correct technique in the published reports of visual complications; many were done on asleep patients, with deep injections rather than submucosal, and some with large gauge needles. ${ }^{1}$ Collectively, from case series published by otolaryngologists who routinely performed injections, a mere three visual complications were reported out of 117,669 injections (0.003\%). All of these disturbances resolved spontaneously, yielding a pristine $0 \%$ rate of permanent complications from experienced providers. Still however, interest in reviving the procedure remains nearly non-existent. It's intriguing that nasal injections of cosmetic fillers are still commonly done despite similar experiences with visual complications. In fact, of all the routine cosmetic filler injection sites, nasal injections have been reported to carry the highest risk of blindness. ${ }^{3}$ Does it make sense that some providers will routinely accept a risk of blindness for a cosmetic procedure, while others won't even consider an analogous treatment for quality of life-impacting allergies?

A cursory review of steroid pharmacology provides additional insights. An overwhelming majority of the intranasal injections performed in the mid-20th century were done using triamcinolone, methylprednisolone 
or other "particulate" steroid formulations. The term "particulate" refers to the tendency of the steroid molecules to bind together, forming microscopic yet mass-occupying collections that can impart a prolonged treatment effect. When one of these clumps of steroid enters choroidal or retinal vessels via collateral vasculature in the intranasal space, a vision-threatening thromboembolism can result. Such is the widely agreed-upon theory of how intranasal injections caused episodes of blindness. ${ }^{1,2}$

What about dexamethasone? This newer-age steroid is ubiquitous and widely used on an international level by providers of nearly all specialties. Importantly, dexamethasone is a non -particulate formulation; that is, it does not have the propensity to form mass-occupying microscopic collections. As such, one could argue a theoretical non-existent risk of visual deficits from an intranasal dexamethasone injection. The medication simply doesn't have the pharmacologic property that underlies the mechanism of the complication. As time goes by, the intranasal cavity stands taller and taller on an increasingly shorter list of anatomic sites into which dexamethasone is NOT routinely injected.

A look over the shoulder of our subspecialist colleagues adds more to the story. Orthopedists and neurosurgeons had more than their fair share of particulate steroid-related complications: There are numerous case reports and series describing permanent paralyses following spinal injections. In contrast to what happened in otolaryngology, these providers continued to implement the highly effective treatment, oftentimes making a switch to non-particulate formulations. To date, there have been no reports of paralyses with nonparticulate injections. Furthermore, a recent meta-analysis found no difference between symptom control for particulate vs. non-particulate spinal injections, thus suggesting that the safety benefit of non-particulates can be achieved without sacrificing effectiveness. ${ }^{4}$ Opthalmologists will not uncommonly administer steroids directly to the intra-vitreal space; and there have yet to be issues with significant adverse events. There's even an FDA improved dexamethasone-eluting implant that's placed within the globe. Multiple prior studies have shown no issues with pathologically increased intra-ocular pressures or any other type of significant visual complication. ${ }^{5,6}$ If dexamethasone can be safely implanted within the actual globe, how worried do we need to be about ophthalmologic complications of a submucosal intranasal injection?

Steroids aside, there's really minimal interest among otolaryngologists to inject any medication intranasally aside from pre-operative lidocaine and epinephrine. Botox, for example, has been shown to be both safe and effective for refractory rhinitis in multiple small series. ${ }^{7}$ This treatment could be viewed as a chemical vidian neurectomy, without the risk of permanent anhydrosis that has kept the surgery from mainstream practice. Despite the promise that's been shown, intranasal botox remains nowhere near as popular as it is for the treatment of salivary glands or cervicofacial dystonias for example. Many anti-histamines are available in intravenous form, has anyone thought of giving those a try? What about a neuromodulator like gabapentin? Sure, injections of these medications may not be the answer for our refractory allergy patients. But the fact that these simple measures have never even been tried over the years despite an established burden of disease is highly suggestive of a purposeful aversion. This avoidance truly may not be evidence-based. We're amidst an explosion of office rhinology procedures driven by costly, sophisticated devices such as sinus and eustachian tube balloons, stents of various kinds, and other nasal implants, many of which have a debatable effectiveness. These are industry-promoted, revenue-driven products. It would be a shame if a simple and historically effective procedure-a simple intranasal injection, remained sidelined from the otolaryngology practice as a result of misperceptions.

\section{References}

1. Mabry RL. Visual loss after intranasal corticosteroid injection. Incidence, causes, and prevention. Arch Otolaryngol. 1981;107(8):484-6.

2. Moss WJ, Kjos KB, Karnezis TT, Lebovits MJ. Intranasal steroid injections and blindness: our personal experience and a review of the past 60 years. Laryngoscope. 2015;125(4):796-800.

3. Beleznay K, Carruthers JDA, Humphrey S, Carruthers A, Jones D. Update on Avoiding and Treating Blindness From Fillers: A Recent Review of the World Literature. Aesthet Surg J. 2019;39(6):662-674. 
4. Feeley IH, Healy EF, Noel J, Kiely PJ, Murphy TM. Particulate and non-particulate steroids in spinal epidurals: a systematic review and meta-analysis. Eur Spine J. 2017 Feb;26(2):336-344.

5. Massa H, Georgoudis P, Panos GD. Dexamethasone intravitreal implant $\left(\right.$ OZURDEX $\left._{\Re}\right)$ for macular edema secondary to noninfectious uveitis: a review of the literature. Ther Deliv. 2019;10(6):343-351.

6. Valenzuela CV, Liu JC, Vila PM, Simon L, Doering M, Lieu JEC. Intranasal Corticosteroids Do Not Lead to Ocular Changes: A Systematic Review and Meta-analysis. Laryngoscope. 2019;129(1):6-12.

7. Zhang EZ, Tan S, Loh I. Botolinum toxin in rhinitis: Literature review and posterior nasal injection in allergic rhinitis. Laryngoscope. 2017;127(11):2447-2454.

Figure I: Intranasal injection (Reproduced with permission)

\section{Key Points :}

1) Intranasal steroid injections were historically very effective, but fell out of favor due to devastating visual complications

2) Other specialties, such as orthopedists and neurosurgeons, had analogous, severe complications with particulate steroids

3) Thromboembolic complications can be avoided by using non-particulate steroids such as dexamethasone

4) To date, there has yet to be any significant attempt to evaluate the safety and effectiveness of intranasal dexamethasone treatments

5) The otolaryngologists' aversion to intranasal injection treatments may not be evidence-based

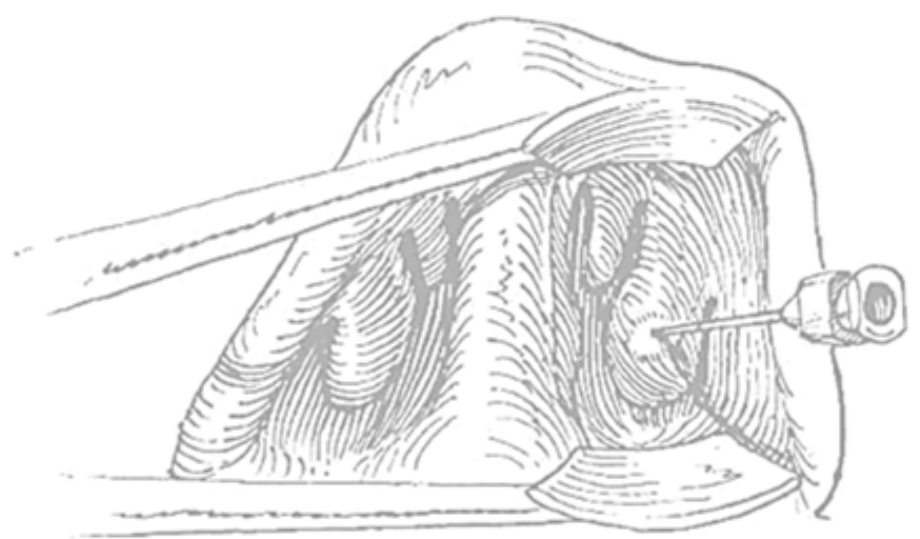

Fig. 1. Irject on just benectn mucosa ef anterior tip of nfencr "uroino*c. Droducing blanching os

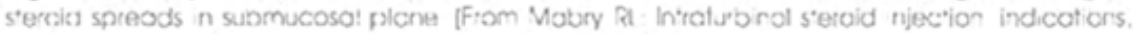
results. and complcations South $\mathrm{Nad}$ J 71:789.791, 1978.) 